\title{
Change of parameters of semiinsulated undoped GaAs nonstoichiometric crystals under annealing
}

\author{
S.V. Shutov ${ }^{1}$, A.D. Shtan'ko ${ }^{2}$, V.V. Kurak ${ }^{2}$, M.B. Litvinova ${ }^{3}$ \\ ${ }^{I} V$. Lashkaryov Institute of Semiconductor Physics \\ 41, prospect Nauky, 03028 Kyiv, Ukraine, \\ Fax: 38(0552)515457,e-mail: shutov_sv@mail.ru \\ ${ }^{2}$ Kherson National Technical University, 24, Beryslavske Sh., 73008 Kherson, Ukraine \\ Phone: 38(0552) 515457, 38(0552) 516468 \\ ${ }^{3}$ National University of Shipbuilding, 44, prospect Ushakova, 73022 Kherson, Ukraine \\ Fax: 38(0552)263118,e-mail:lmb@ist.com.ua
}

\begin{abstract}
The time dependences of changes of the electrophysical, mechanical, and light emitting characteristics of semiinsulated undoped GaAs single crystals on the dissolving annealing $\left(T=1050{ }^{\circ} \mathrm{C}\right)$ with the consequent quenching are obtained. The role of vacancies, interstitial point defects, and dislocations in nonstoichiometric crystals is revealed.
\end{abstract}

Keywords: gallium arsenide single crystal, annealing, point defect, vacancy.

Manuscript received 25.05.07; accepted for publication 19.12.07; published online 31.01.08.

\section{Introduction}

Last time, the annealing of semiinsulated undoped (SIU) GaAs crystals under temperature higher than $1000{ }^{\circ} \mathrm{C}$, called in [1] as "dissolving annealing", is widely used in two-stage technological heat treatment (HT) of this material. Some works, such as [1-3], represent data on annealing conditions of crystals with similar, at the first sight, initial characteristics. However, the results obtained after HT in different works differ essentially from one another.

In this work, the study of the dissolving annealing at $1050{ }^{\circ} \mathrm{C}$ of SIU GaAs crystals with different vacancy compositions was carried out. After HT, the samples were subjected to fast cooling (quenching) that led to the "freezing" of point defects. As a result, the concentration of point defects was close to that at the annealing temperature.

\section{Experimental}

Samples with size $\approx 4 \times 2 \times 1 \mathrm{~mm}$ were used for HT. These samples were cut from Czochralski-grown SIU GaAs (100) single crystals with the $n$-type of conductivity. In such crystals, it is possible to estimate the concentration of vacancies and interstitial defects (gallium vacancies $\left[\mathrm{V}_{\mathrm{Ga}}\right]$, arsenic vacancies $\left[\mathrm{V}_{\mathrm{As}}\right]$, and interstitial arsenic $\left[\mathrm{As}_{\mathrm{i}}\right]$ ) using photoluminescence (PL) spectra and data on mechanical stresses $\sigma$, respectively $[4,5]$.
At the initial stage of experiments (before HT), the samples had the $n$-type of conductivity, specific resistance $\rho=7 \cdot 10^{7}-2 \cdot 10^{8} \mathrm{Ohm} \cdot \mathrm{cm}$, and the density of dislocations $N_{d}=3 \cdot 10^{4}-6 \cdot 10^{5} \mathrm{~cm}^{-2}$. All samples were separated in two groups: group I included the crystals obtained with gallium surplus, where dominating point defects are arsenic vacancies and the lattice constant is smaller than that in stoichiometric crystals; group II included the crystals obtained with arsenic surplus, where dominating point defects are interstitial arsenic atoms and the lattice constant is greater than that in stoichiometric crystals [5-7].

HT of samples was carried out in the atmosphere of arsenic vapor into ampoules with a useful volume of $\sim 4 \mathrm{~cm}^{-3}$ evacuated up to the pressure $<10^{-3} \mathrm{~Pa}$. HT duration $(t)$ was from $10 \mathrm{~min}$ up to $24 \mathrm{~h}$. To create the equilibrium pressure of arsenic vapor, arsenic was preformed with a mass of $20 \mathrm{mg}$ and was placed into ampoules. After HT, the removal of disturbed surface layers with the thickness more than $100 \mu \mathrm{m}$ was realized by using, at first, mechanical polishing and then chemical polishing in $\mathrm{H}_{2} \mathrm{SO}_{4}: \mathrm{H}_{2} \mathrm{O}_{2}: \mathrm{H}_{2} \mathrm{O}$ (3:1:1).

PL spectra were measured by standard procedure [8]. As a source for PL excitation, a helium-neon laser with the wavelength $\lambda=0.63 \mu \mathrm{m}$ was used. The minority carrier lifetime was determined by the luminescence method reviewed in [8] in detail. Specific resistance and carrier mobility at $300 \mathrm{~K}$ were measured by the Hall method, and the concentration of EL2 
centers was determined by measuring the optical absorption of radiation with $\lambda=1 \mu \mathrm{m}$ [9]. Mechanical stresses $\sigma$ were determined by the optical polarization method at the wavelength $\lambda=1.15 \mu \mathrm{m}$ by using of a Senarmon compensator [10].

If the HT duration $t$, during which changes of the parameters under investigation were observed, was kept in the initial interval $t<2-3 \mathrm{~h}$, and the subsequent increase of HT duration had not influence the parameters, then experimental results were considered only in the mentioned time interval.

\section{Results}

Small samples of sizes $\sim 4 \times 2 \times 1 \mathrm{~mm}$ give us a possibility to ignore the inhomogeneity of the distribution of intrinsic point defects (IPD) along sample cross sections. Moreover, we think that, due to the surface relaxation of residual mechanical stresses, the stresses $\sigma$ in the initial crystals, first of all, are caused by a deviation of the lattice constant from the stoichiometric one [6]. After annealing, crystals of both groups kept the $n$-type of conductivity and the initial values of the dislocation density. At the same time, changes of the electrophysical and luminescent properties for the first and the second groups of crystals (Figs. 2-5) had different characters.

Fig. 1 (curve 1) represents the short-wave part of typical PL spectra of SIU GaAs single crystals. These spectra show two radiation bands - edge band (a) with the intensity $I_{1}$ and impurity band (b) with the intensity $I_{2}$ which are caused by radiative transitions through the acceptor level created by carbon at the place of an arsenic vacancy [11].

In PL spectrum of group I of crystals, a drop of the edge band intensity was observed with increase in the HT duration [Fig. 2(3)], and a radiation band with the energy $h v_{m}=1.480 \mathrm{eV}$ appeared [Fig. 1(2)]. The ratio of intensities $I_{2} / I_{1}(t)$ monotonously increased [Fig. 2(2)], and a position of the PL edge band shifted to lower energies [Figs 1(2) and 2(1)].

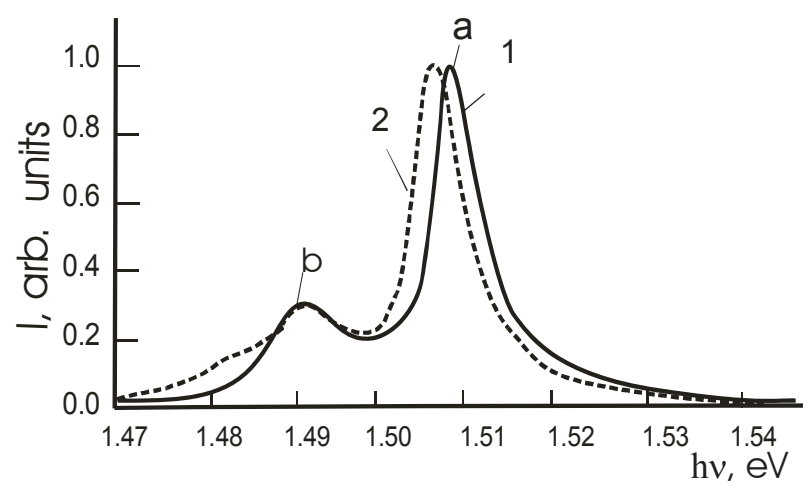

Fig. 1. PL spectrum of crystals (group I) at $77 \mathrm{~K}: 1$ - before HT; 2 - after HT during $40 \mathrm{~min}$.

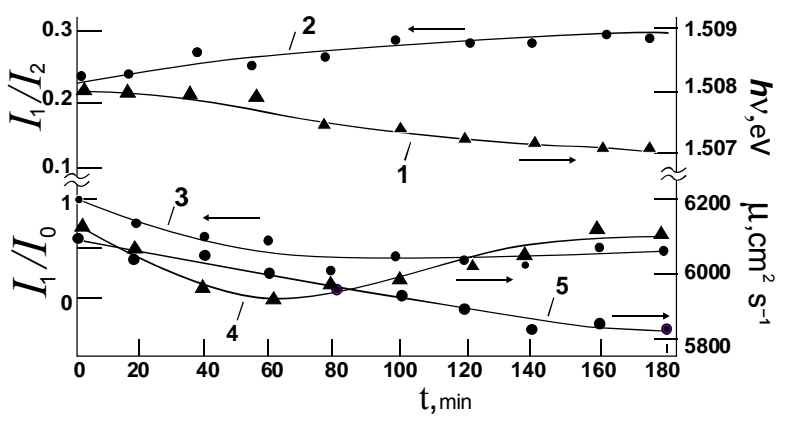

Fig. 2. Changes after HT for the crystals of groups I (1-4) and II (5): 1 - energy of the edge PL band maximum; 2 - ratio of the intensity of the impurity PL band to that of the edge PL band; 3 - relative intensity of the edge PL band $\left(I_{0}\right.$ - intensity for the initial crystal); 4, 5-majority carrier mobility.

Initial stresses $\sigma$ in this group of crystals were negative. The increase of $\sigma$ up to positive values was observed during the first hour of HT. After that, values of $\sigma$ gradually reduced (see the insert in Fig. 3), so the character of $\sigma$ became negative at $t>17 \mathrm{~h}$ again [Fig. 3(1)]. During the first $60-70 \mathrm{~min}$ of HT, the mobility of major carriers reduces, then passes to the extreme point, and increases closely to the initial value at $t>70 \mathrm{~min}$ [Fig. 2(4)]. The decrease of the specific resistance $\rho(t)$ was observed at $t>1 \mathrm{~h}$ [see Fig. 5(2) and the insert in Fig. 5]. The concentration of EL2 centers $N_{\mathrm{EL} 2}(t)$ [Fig. 4(5)] and the concentration of major carriers [Fig. 4(3)] monotonically increase, and the minority carrier lifetime $\tau(t)$ decreases [Fig. 5(4)].

For group II of crystals, the gradual decrease of the edge band PL intensity took place during $17-18 \mathrm{~h}$ of HT (Fig. 4(1)). At $t>18 \mathrm{~h}$, the value of $I_{1}(t) / I_{0}\left(I_{0}-\right.$ edge band PL intensity before HT) is stabilized. The ratio of intensities $I_{2} / I_{1}(t)$ [Fig. 4(2)] and the energy position of the edge band maximum did not change with increase in $t$.

In the initial samples of group II, mechanical stresses were positive. As a result of annealing, the decrease in $\sigma$ with increase in $t$ was observed initially. Then, at $t>19 \mathrm{~h}$, values of $\sigma$ gradually increased

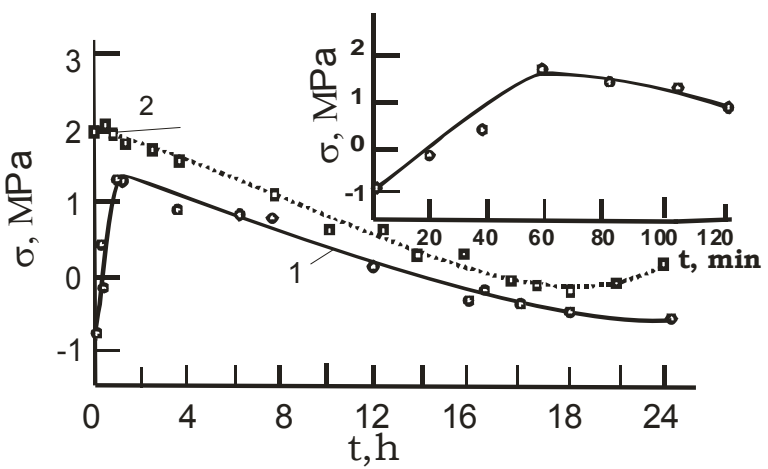

Fig. 3. Changes of mechanical stresses in the crystals of groups I (1) and II (2) $\left(N_{d}=(1-2) \cdot 10^{5} \mathrm{~cm}^{-3}\right)$. In the insert: change of mechanical stresses in the crystals of group during the two-hour initial period of HT. 


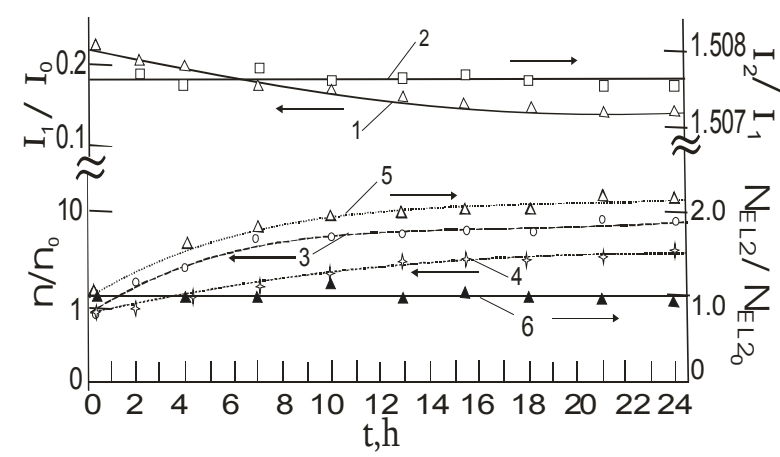

Fig. 4. Changes for the crystals of groups I $(3,5)$ and II $(1$, $2,4,6): 1$ - relative intensity of the edge PL band $\left(I_{0}-\right.$ intensity for the initial crystal); 2 - ratio of the intensity of the impurity PL band to that of the edge PL band; 3, 4 relative concentration of majority carriers $\left(n_{0}-\right.$ concentration in the initial crystal); 5,6 - relative concentration of EL2 centers (EL2 $2_{0}$ - concentration in the initial crystal).

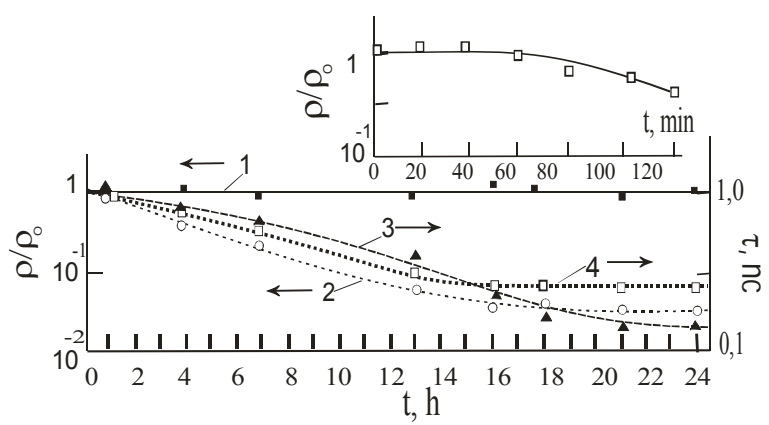

Fig. 5. Changes for the crystals of groups I $(2,4)$ and II $(1,3)$ : 1,2 - specific resistance ( $\rho_{0}-$ value in the initial crystal); 3, 4 - minority carrier lifetime. In the insert: change of the specific resistance in the crystals of group I during the twohour initial period of HT.

[Fig. 3(2)]. The decrease of the minority carrier lifetime [Fig. 5(3)] and the major carrier mobility [Fig. 2(5)] were also observed with increase in $t$. At the same time, the value of $n / n_{0}$ increased with the HT duration [Fig. 4(4)]. The concentration of EL2 centers [Fig. 4(6)] and the specific resistance $\rho$ of crystals [Fig. 5(1)] did not change essentially. The value of dislocation density had no essential influence on the parameters of annealed crystals of both groups.

\section{Discussion}

\subsection{Change of mechanical stresses}

Interstitial defects in GaAs crystals result in increasing the lattice constant $\alpha$, which corresponds to tensile stresses (positive). The presence of vacancy defects leads to a decrease in $\alpha$, which corresponds to compression stresses (negative). Accordingly, the increase or decrease in mechanical stresses $\sigma$ reflects the increase of the interstitial or vacancy IPD concentration and gives us a possibility to make conclusion about a change of the defect structure of crystals after HT [5]. Therefore, first of all, we will carry out the analysis of the change in $\sigma$ in both groups of samples.

Negative values of $\sigma$ in initial crystals of group I are caused by the predominance of nonassociated vacancy defects over interstitial ones, whereas positive values of $\sigma$ in crystals of group II are related to the predominance of nonassociated interstitial defects over vacancy ones $[5,6]$. The well-known results of radiographic and metallographic investigations of the influence of various HT conditions on the formation of microdefects (MD) in undoped GaAs single crystals [1] allow one to explain the character of the dependences $\sigma=f(t)$ obtained in this work in the following way.

Abrupt cooling of samples leads to the IPD "freezing", and the IPD concentration in a crystal at $T=300 \mathrm{~K}$ approaches the equilibrium one at the HT temperature. A surplus atomic component is present in initial crystals of both groups $[5,6]$. For the crystals of group I, it is gallium. In samples before HT, it has high stage of association in the form of microdefects; therefore, nonassociated arsenic vacancies are predominant IPD [1,6]. The data given in [1] have shown that the decay of associates on interstitial atoms $\mathrm{Ga}_{i}$ during the process of dissolving annealing at temperatures higher than $1000{ }^{\circ} \mathrm{C}$ takes place. It increases positive stresses in crystals during the first hour of HT. Along with the MD decay, the dissolution of near-dislocation atmospheres [1] and the diffusion of predominant IPD in the sample volume are realized. It is known that near-dislocation atmospheres have complex structure composed of alternating vacancy and interstitial defects [6]. However, the same IPD prevail in their structure, as in the crystal volume; in this case they are arsenic vacancies [6]. The increase of their concentration results in a gradual decrease of $\sigma$. Finally, $\sigma$ becomes negative.

Evidently, the change of stresses with increase in the HT time in crystals of group II [Fig. 3(2)] also deals with IPD diffusion from predislocation atmospheres. The dominating defect in such crystals is interstitial arsenic [6], but $\mathrm{V}_{\mathrm{Ga}}$ is also present in predislocation atmospheres and its concentration prevails in the $\mathrm{V}_{\mathrm{As}}-\mathrm{V}_{\text {Ga }}$ pair.

Let's consider the diffusion of $\mathrm{V}_{\mathrm{Ga}}$ and $\mathrm{As}_{i}$ from dislocations. According to the expressions for diffusivity (D) [12], we have following results at $T=1050^{\circ} \mathrm{C}$ : $D\left(\mathrm{As}_{i}\right) \approx 8 \cdot 10^{-13} \mathrm{~cm}^{2} / \mathrm{s}, D\left(\mathrm{~V}_{\mathrm{Ga}}\right) \approx 3 \cdot 10^{-11} \mathrm{~cm}^{2} / \mathrm{s}$. The value of $D\left(\mathrm{~V}_{\mathrm{Ga}}\right)$ is an order greater than the value of $D\left(\mathrm{As}_{i}\right)$. As a result, the leading (with respect to $\mathrm{As}_{i}$ ) diffusion of gallium vacancies in the crystal volume occurs. This leading diffusion decreases positive stresses, as it is shown in Fig. 3(1). With the further increase of the HT time, the "lagging" diffusion of $\mathrm{As}_{i}$ atoms in the crystal volume causes the growth of $\sigma$. This assumption can be confirmed with the calculations as follows.

The distance $l$, on which $\mathrm{V}_{\mathrm{Ga}}$ can move during the time $t$, can be found from the formula

$l=\left[D\left(\mathrm{~V}_{\mathrm{Ga}}\right) t\right]^{1 / 2}$. 
The radius of the area related to the influence of a separate dislocation is determined as a half distance between dislocations [6]

$r=0.5 N_{d}^{-1 / 2}$

(for the sample with the $N_{d}=1 \cdot 10^{5} \mathrm{~cm}^{-2}, r \approx 15 \mu \mathrm{m}$ ). When vacancies of gallium diffusing from one dislocation achieve the "area of influence" of another one, the size $l$ is equal to $r$. That is, there will be a distribution of vacancies throughout the crystal volume. Let's find the time necessary for such a process. Relations (1) and (2) and the condition $l=r$ yield

$\left[D\left(\mathrm{~V}_{\mathrm{Ga}}\right) t\right]^{1 / 2}=0.5 N_{d}^{-1 / 2}$,

whence we get

$t=0.25\left[D\left(\mathrm{~V}_{\mathrm{Ga}}\right) \cdot N_{d}\right]^{-1}$.

As seen from Fig. 3(1), the reduction of $\sigma$ occurs during $t \approx 18 \mathrm{~h}$. In expression (3), such value of $t$ corresponds to $N_{d} \approx 1.3 \cdot 10^{5} \mathrm{~cm}^{-2}$, which is in agreement with the values of dislocation density in the crystals under investigation $\left[N_{d} \approx(1-2) \cdot 10^{5} \mathrm{~cm}^{-2}\right]$.

Interstitial arsenic diffuses during $t \approx 18 \mathrm{~h}$ at a distance $l_{\mathrm{As}} \approx 2 \mu \mathrm{m}$ from a dislocation [according to expression (1)]. This distance is significantly lower than $r \approx 15 \mu \mathrm{m}$. By the time moment when gallium vacancies fill the whole volume of a crystal, the influence of the growth in the interstitial arsenic concentration on $\sigma$ becomes apparent only. That is why a decrease of stresses is observed till $t \approx 18 \mathrm{~h}$, and, at $t>18 \mathrm{~h}$, the increase of mechanical stresses occurs.

\subsection{Change of radiant and electrophysical characteristics}

During HT, the dissolution of predislocation atmospheres, which have mainly arsenic vacancies in the first group of crystals, leads to an increase of the concentration of nonassociated $\mathrm{V}_{\mathrm{As}}$ in the crystal volume. Accordingly, the concentration of easily ionizable dopants in the arsenic sublattice, in particular $C_{\mathrm{VAs}}$, is increased. This is indicated by the increase in $I_{2} / I_{1}(t)$ [Fig. 2(2)]. The appearance of a PL band with $h v_{m}=1.480 \mathrm{eV}$, which is determined by radiative transitions through the $\mathrm{Si}_{\mathrm{VAs}}$ acceptor level [11], is the evidence of the thermal activation of these centers during HT. As was shown in [13], an increase in the total concentration of $\left[\mathrm{Si}_{\mathrm{VAs}}\right]+\left[C_{\mathrm{VAs}}\right]$ corresponds to a reduction of the energy of the edge PL band maximum observable in Fig. 2(1).

A reduction of the edge $\mathrm{PL}$ band intensity with increase in $t$ [Fig. 2(3)] in crystals of group I should be connected with increase in the concentration of nonradiative recombination centers, which is confirmed by a decrease of the minority carrier lifetime [Fig. 5(4)].
A reduction of the edge PL band intensity in crystals of group II [Fig. 4(1)] is caused by the same reason, by the increase of the number of nonradiative recombination centers during HT which is affected by a decrease of the minority carrier lifetime [Fig 5(3)]. As seen from Fig. 5, the decrease in $\tau(t)$ is more essential in the second group of crystals, than that in the first group. This is caused by the movement of gallium vacancies from near-dislocation atmospheres into the crystal volume, and, as it is well known, gallium vacancies are the centers of nonradiative recombination [9]. In addition, no change of the $I_{2} / I_{1}(t)$ ratio is observed in crystals of group II (the decrease of $\tau(\mathrm{t})$ affects equally $I_{2}$ and $I_{1}$ ) [see Fig. 4(2)]. This implies that the concentration of $C_{\mathrm{VAs}}$ centers is constant, and a corresponding displacement of the energy position of the edge PL band, as a result of HT, does not occur [13].

A decrease of the majority carrier mobility $\mu(t)$ in crystals of group I during the first hour of HT [Fig. 2(4)] is related to the increase in the concentration of nonassociated IPD in the crystal volume. However, the increase of $\mu$ is observed at $t>60 \mathrm{~min}$. Most probably, it is caused by the following reason. It is known [6] that dislocations with the edge component have a disseminating influence on charge carriers, by reducing their mobility. At that, near-dislocation atmospheres play an essential role. Their resorption during HT reduces the influence of dislocations. Apparently, the dissipative influence of near-dislocation atmospheres is higher, than the influence of nonassociated arsenic vacancies in the crystal volume. Therefore, the carrier mobility increases with disappearance of atmospheres. Really, the extremum of the curve $\mu(t)$ [Fig. 2(4)] for the given group of crystals coincides with a maximum of $\sigma(t)$ (see the insert in Fig. 2), and the decrease of the mobility corresponds to the decrease of $\sigma(t)$ caused by resorption of neardislocation atmospheres as a result of the diffusion of $\mathrm{V}_{\mathrm{As}}$ into the crystal volume.

In crystals of group II, a decrease in the disseminating influence of atmospheres on charge carriers does not "compensate" the increasing influence of nonassociated IPD (gallium vacancies and interstitial arsenic). Therefore, a gradual decrease in $\mu(t)$ is observed [Fig. 2(5)]. Differences in the characters of $\mu(t)$ dependences for crystals of groups I and II confirm that a change in the composition of near-dislocation atmospheres (prevalence of gallium or arsenic vacancies) has influence on a change of the carrier's mobility during HT.

The change of the specific resistance in crystals of both groups after HT occurs, by depending on the change of $n(t)$ and $\mu(t)$. During the first hour of HT, the concentration of charge carriers in crystals of group I increases [Fig. 4(3)] as a result of the increase in the concentration of donor EL2 centers [Fig. 4(5)]. During the same time, the values of carrier's mobility are reduced. According to the relation

$\rho \sim(\mu n)^{-1}$ 
values $\rho(t) \approx$ const (see the insert in Fig. 5). At $t>1 \mathrm{~h}$, $\mu(t)$ and $n(t)$ increase, and so the values of $\rho(t)$ decrease [Fig. 5(2)].

As a result of $\mathrm{HT}, N_{\mathrm{EL} 2}(t)$ does not change in crystals of group II, while the concentration of charge carriers increases. Hence, there is the thermal activation of other donor centers. They are, most probably, $\mathrm{Si}_{\mathrm{Ga}}$ [9]. Because $n(t)$ increases and $\mu(t)$ reduces, $\rho(t)$ is not changed essentially according to relation (4) [Fig. 5(1)].

\section{Conclusions}

We have established a nonmonotonic character of the dependences of the parameters for semiinsulated undoped GaAs crystals on the time of heat treatment under conditions of quenching. The obtained facts are caused by the thermal redistribution of own and impurity point defects under dissolving annealing. Essential differences in the dependences of the parameters are revealed for crystals of two groups grown with surplus of gallium or arsenic.

\section{References}

1. B.T. Bublik, M.I. Voronova, A.V. Markov, K.D. Scherbachov // Crystallography Reports 45(5), p. 893-898 (2000).

2. Z.-Q. Fang, G.C. Reynolds, D.C. Looc, N.G. Paraskevopoulos, T.E. Anderson, R.L. Jones // J. Appl. Phys. 83 (1), p. 260-265 (1998).
3. N.M. Litovchenko, A.V. Prokhorovich, O.N. Stril'chuk et al. // Optoelectronika Poluprov. Tekhnika 38, p. 302-305 (2003) (in Russian).

4. V.F. Kovalenko, M.B. Litvinova, V.A. Krasnov // Ibid. 37, p. 202-208 (2002) (in Russian).

5. M.B. Litvinova, A.D. Shtan'ko // Ibid. 40, p. 228233 (2005) (in Russian).

6. M.G. Mil'vidskii, V.B. Osvennskii, Structural Defects in Semiconductor Single Crystals. Metallurgiya, Moscow, 1984 (in Russian).

7. V.V. Voronkov, A.U. Bol'sheva, R.I. Glorizova, L.I. Kolesnik, O.G. Stolyarov // Crystallography Reports 32(1), p. 208-212 (1987).

8. G.P. Peka, V.F. Kovalenko, V.N. Kutsenko, Luminescent Methods of Control over the Parameters of Semiconductor Materials and Devices. Tekhnika, Kyiv, 1986 (in Russian).

9. K.D. Glinchuk, V.I. Hyroshev, A.V. Prokhorovich // Optoelectronika Poluprovodnikovaya Tekhnika 24, p. 66-86 (1992) (in Russian).

10. V.I. Nikitenko, Strain change in flat junctions of silicon with metals / In: Strain and Dislocations in Semiconductors. Acad. of Sci. of USSR, Moscow, 1962 (in Russian).

11. K.D. Glinchuk, N.M. Litovchenko, A.V. Prokhorovich, O.N. Stril'chuk // Semiconductors 35(4), p. 384-386 (2001).

12. F.S. Shishiyany, Diffusion and Degradation in Semiconductor Materials and Devices. Stiintsa, Kishinev, 1978 (in Russian).

13. K.D. Glinchuk, A.V. Prokhorovich, F.M. Vorobkalo // Cryst. Research Technol. 31(8), p. 1045-1049 (1996). 